\title{
A High-Precision Control Scheme Based on Active Disturbance Rejection Control for a Three-Axis Inertially Stabilized Platform for Aerial Remote Sensing Applications
}

\author{
Xiangyang Zhou $\mathbb{D}^{1,2}$ Chao Yang, ${ }^{1}$ Beilei Zhao, ${ }^{1}$ Libo Zhao, ${ }^{2}$ and Zhuangsheng Zhu $\mathbb{D}^{1}$ \\ ${ }^{1}$ School of Instrumentation Science and Opto-electronics Engineering, Beihang University (BUAA), Beijing 100191, China \\ ${ }^{2}$ State Key Laboratory for Manufacturing Systems Engineering, Xi'an Jiaotong University, Xi'an 710049, China
}

Correspondence should be addressed to Xiangyang Zhou; xyzhou@buaa.edu.cn and Zhuangsheng Zhu; zszhu@buaa.edu.cn

Received 1 December 2017; Revised 12 February 2018; Accepted 5 April 2018; Published 17 July 2018

Academic Editor: Biswajeet Pradhan

Copyright (@ 2018 Xiangyang Zhou et al. This is an open access article distributed under the Creative Commons Attribution License, which permits unrestricted use, distribution, and reproduction in any medium, provided the original work is properly cited.

\begin{abstract}
This paper presents a high-precision control scheme based on active disturbance rejection control (ADRC) to improve the stabilization accuracy of an inertially stabilized platform (ISP) for aerial remote sensing applications. The ADRC controller is designed to suppress the effects of the disturbance on the stabilization accuracy that consists of a tracking differentiator, a nonlinear state error feedback, and an extended state observer. By the ADRC controller, the effects of both the internal uncertain dynamics and the external multisource disturbances on the system output are compensated as a total disturbance in real time. The disturbance rejection ability of the ADRC is analyzed by simulations. To verify the method, the experiments are conducted. The results show that compared with the conventional PID controller, the ADRC has excellent capability in disturbance rejection, by which the effect of main friction disturbance on the control system can be weakened seriously and the stabilization accuracy of the ISP is improved significantly.
\end{abstract}

\section{Introduction}

It is of crucial importance for an aerial remote sensing system to obtain the high-resolution images under a nonideal multisource disturbance environment. The inertially stabilized platform (ISP) is a key component for an aerial remote sensing system, which is used to hold and control the line of sight (LOS) of the imaging sensors keeping steady relative to the tracking object in the inertial space $[1,2]$. If the highresolution images of the imaging sensor were obtained, the ISP should have the capability to effectively isolate the attitude changes of aircraft in three axes; besides, it is required to suppress the multisource disturbances whatever inside or outside of the aircraft body so that the high control precision can be gotten. Therefore, the most critical performance metric for the ISP is disturbance rejection.

Generally, the ISP is placed between the aircraft and imaging sensor served as a physical interface. It is a principal issue for the control system of the ISP to minimize the effects of disturbances introduced on the ISP [1]. In [2], a proportional integral control scheme is proposed to realize zero steady-state error of angular output when facing disturbances from carrier. In [3], a hybrid adaptive fuzzy proportionalintegral-derivative (PID) controller is developed for a servo system with nonlinear property and uncertainties. In [4], a new current loop inside rate loop is particularly designed to suppress the influences of voltage fluctuation and motor back electromotive force on control precision. In [5], the model uncertainties and unmeasurable disturbances existing objectively are solved by introducing internal model control for a two-axis ISP system. In [6], an approach to compensate disturbing torque based on acceleration feedforward for the three-axis ISP is proposed. In [7], a parameter identification method for LuGre model based on multilevel coordinate search algorithm was presented. In [8], a compound control scheme based on both of the model reference adaptive control (MRAC) and PID control methods is proposed. 


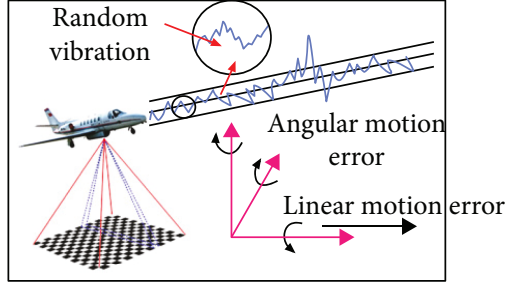

External disturbance: gust, turbulance, and so on Internal disturbance: engine vibration and so on

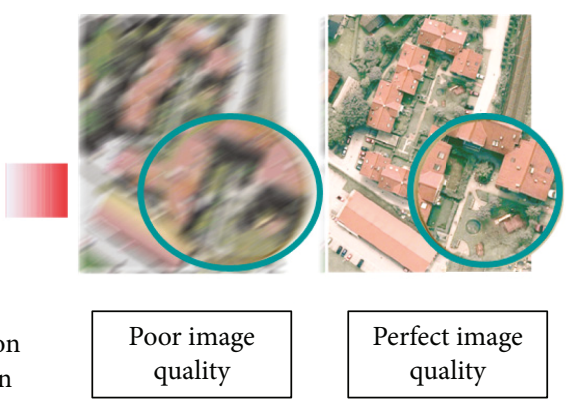

FIGURE 1: Schematic diagram: effect of the ISP on improving the images quality in an aerial remote sensing system.

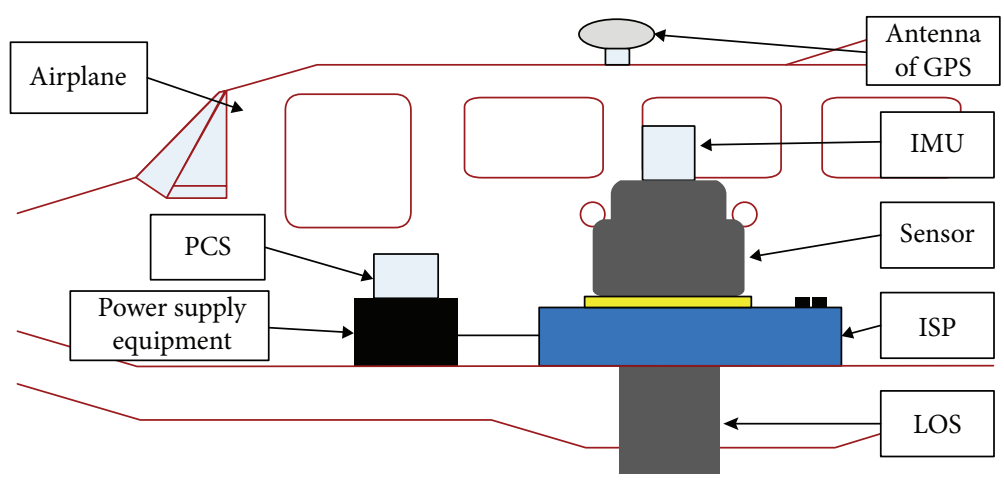

Figure 2: Schematic diagram of an aerial remote sensing system.

The active disturbance rejection control (ADRC) was proposed to deal with the nonlinear systems, where both uncertain dynamics and disturbances exist. The ADRC is composed of three components, that is, a tracking differentiator (TD), a nonlinear state error feedback (NLSEF), and an extended state observer (ESO). The key in the ADRC is to online estimate the total uncertainty, which lumps the internal uncertain dynamics and the external disturbances by the ESO. The ADRC can solve quickly and effectively the problems of nonlinear, strong coupling, and so on $[9,10]$. The ADRC not only can improve the dynamic performance of the system but also can achieve good adaptability and robustness $[11,12]$. A large number of simulation experiments show that the ADRC algorithm is simple to detect and compensate the disturbance of the control object automatically $[13,14]$. This design methodology requires very little information about the plant dynamics, is very easy to tune, and has a very good disturbance rejection capability. In [15], a feedback linearization based the ESO control law is proposed for the trajectory tracking control of a flexible joint robotic system. In [16], the effectiveness of the ESO in the estimation of the states and uncertainties is demonstrated. In [17], an ADRC for the antenna pointing control of a large flexible satellite system is designed, by which the high pointing accuracy and rotation speed are achieved.

In this paper, to improve the ability of disturbance rejection of an aerial three-axis ISP, a disturbance rejection scheme based on the ADRC is proposed. To verify the method, the simulations and experiments are carried out, respectively. The rest of this paper is arranged as follows.
Section 2 first introduces the background of this work. Section 3 explains the ADRC principle and algorithm that includes three parts: the TD, the NLSEF, and the ESO. Section 4 demonstrates the simulation analysis, including ADRC controller as well as the PID controller. The ADRC experiment is in Section 5, including the static base experiment and the moving base experiment. In the end, a brief conclusion is given in Section 6.

\section{Background}

2.1. Aerial Remote Sensing System. Figure 1 shows a schematic diagram to illustrate the important effect of the ISP on improving the image quality in an aerial remote sensing system. In general, an aerial remote sensing system consists of an aircraft vehicle, an imaging sensor, a three-axis ISP, and a position and orientation system (POS). During the work, the ISP is used as a physical interface to separate the imaging sensor and the aircraft. Depending on the ISP, the serious influences of various disturbances on the imaging sensors are effectively suppressed, by which the LOS can be kept stable all along and the high-resolution images are gotten eventually.

2.2. The Role of Three-Axis ISP System. Figure 2 shows the schematic diagram of an aerial remote sensing system. As shown in this figure, during application, the ISP is installed on the floor of the airplane. Meanwhile, the imaging sensor is installed on the inner azimuth gimbal of the ISP, and the POS is installed on the top of the imaging 


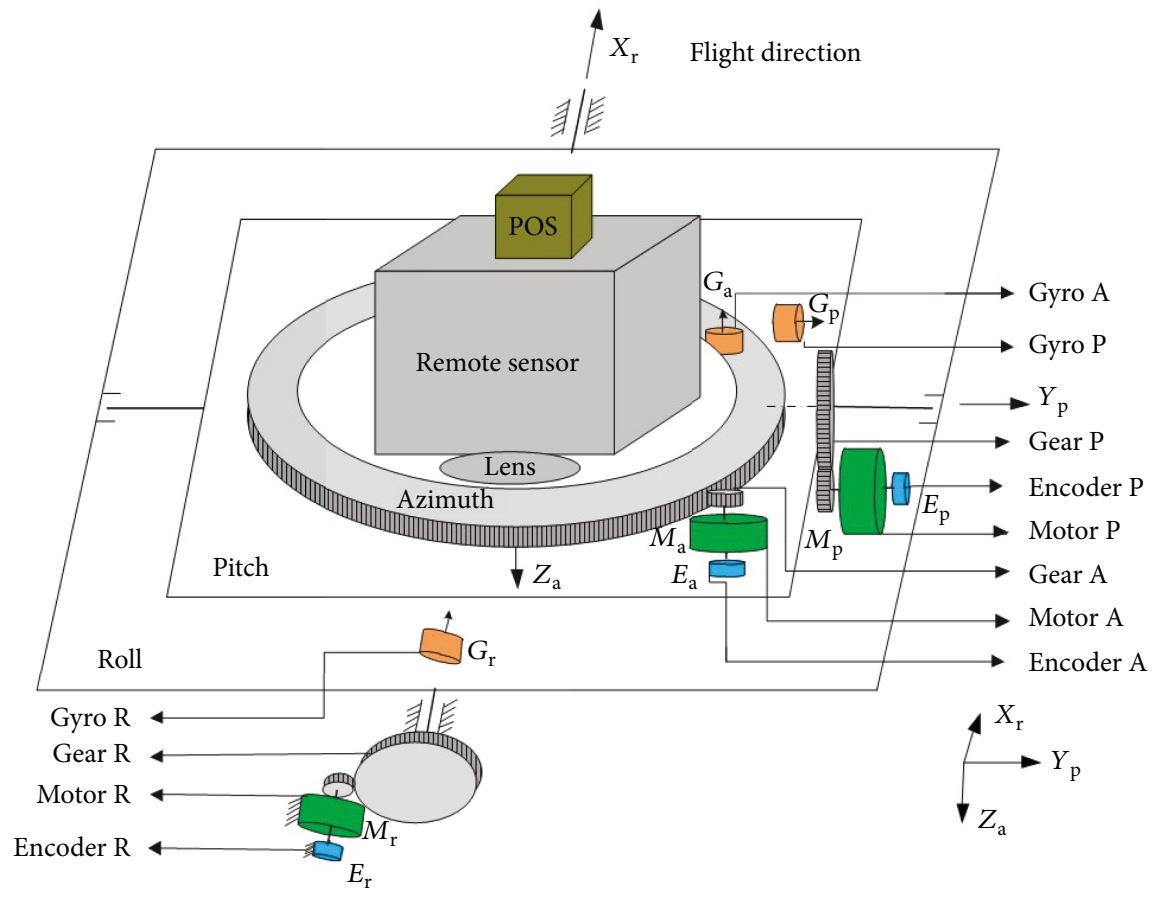

FIgURE 3: Schematic diagram of the three-axis ISP's principle.

sensor. When the aircraft rotates or jitters, the high-precision attitude reference information is measured by the POS in real time, which is then provided to the ISP control system to make the LOS of imaging sensor to achieve the high stabilization precision.

2.3. Working Principle of Three-Axis ISP System. Figure 3 shows the schematic diagram of the three-axis ISP's principle. As shown in the figure, the ISP consists of three gimbals, which are azimuth gimbal (A-gimbal), pitch gimbal (P-gimbal), and roll gimbal (R-gimbal) from the outside to the inside. Three rate gyros installed on the three different gimbals are used to measure inertial angular rate of $\mathrm{P}$-gimbal, R-gimbal, and A-gimbal, which are used to improve the stabilization precision through compensating the difference between the rate command input and the angular rate of the gimbal. On the other aspects, three photoelectric encoders installed on different gimbals are used to measure the relative angular position changes between two adjacent gimbals. Three servo motors installed on different gimbals are used to drive R-gimbal, $\mathrm{P}$-gimbal, and A-gimbal rotating inversely to compensate the attitude changes to three gimbals in real time.

\section{Design of the ADRC Controller}

3.1. Tracking Differentiator (TD). For a second-order system [18], we get

$$
\begin{aligned}
& \dot{z}_{1}=z_{2}, \\
& \dot{z}_{2}=f\left(z_{1}, z_{2}\right),
\end{aligned}
$$

if the solution of (1) satisfies

$$
\begin{aligned}
& \lim _{t \rightarrow \infty} z_{1}(t)=0, \\
& \lim _{t \rightarrow \infty} z_{2}(t)=0 .
\end{aligned}
$$

So, for any boundary integrable variable, $v(t)$, and any constants, $T>0$ and $r>0$, the solution $x_{1}(t)$ of the system

$$
\begin{aligned}
& \dot{x}_{1}=x_{2}, \\
& \dot{x}_{2}=r^{2} \cdot f\left(x_{1}-v(t), \frac{x_{2}}{r}\right)
\end{aligned}
$$

can meet the following relationship:

$$
\lim _{r \rightarrow \infty} \int_{0}^{T}\left|x_{1}(r, t)-v(t)\right| d t=0 .
$$

Therefore, assuming that a function $f\left(z_{1}, z_{2}\right)$ can be found to make (1) asymptotically stable at the origin, a nonlinear tracking differentiator of $v(t)$ in the form of (3) can be constructed, in which the same function $f\left(z_{1}, z_{2}\right)$ is used [19].

Kasim and Riyadh [20] have made some investigations on traditional structures and essential properties of the nonlinear TD. A kind of second-order nonlinear TD based on second-order bang-bang switch system has been proposed:

$$
\begin{aligned}
& \dot{x}_{1}=x_{2}, \\
& \dot{x}_{2}=-r \cdot \operatorname{sign}\left(x_{1}-v_{0}(t)+\frac{x_{2}\left|x_{2}\right|}{2 r}\right),
\end{aligned}
$$

where $x_{1}$ is the desired trajectory and $x_{2}$ is its derivative. Note that the parameter $r$ is an application dependent and it is set 
accordingly to speed up or slow down the transient profile. Then, $x_{2}$ is denoted as the TD of $v_{0}(t)$.

In order to avoid chattering near the origin, changing the sign function to linear saturation function sat, then the modified TD is represented by [21-23]

$$
\operatorname{sat}(x, \delta)= \begin{cases}\operatorname{sign}(x), & |x| \geq \delta, \\ \frac{x}{\delta}, & |x|<\delta .\end{cases}
$$

The TD gets the differentiated signal by computing the integration of the relational viable. So it can efficiently weaken the effect of noise, while the classical differential component enlarges it and the differentiated signal obtained by this way is far from the real one. In some sense, the differential component of the TD is feasible physically, while differential components of the classical methods are not feasible physically [21].

3.2. Nonlinear State Error Feedback (NLSEF). For the firstorder system [22],

$$
\dot{x}=w(x, t)+u \text {. }
$$

$w(x, t)$ is the sum of the perturbations of the system, and linear feedback is used for the control object:

$$
u=-k x, \quad k>0 .
$$

Then, the closed-loop system can be expressed:

$$
x=-k x+w(x, t)
$$

The closed-loop equation of the finishing system is obtained [23]:

$$
\frac{1}{2} \frac{d x^{2}}{d t}=-k x^{2}+x w=-k\left(x-\frac{w}{2 k}\right)^{2}+\frac{w^{2}}{4 k} .
$$

If the state quantity $x$ converges, then $d x^{2} / d t<0$ is needed, that is to say, it needs to be satisfied:

$$
\left|x-\frac{w}{2 k}\right|>\frac{|w|}{2 k} .
$$

It is easy to get

$$
|x|>\frac{|w|}{k}
$$

As long as $|x|>|w| / k$, there must be $d x^{2} / d t<0$. The state parameter $x$ converges, and the system disturbance $w(x, t)$ can also be well suppressed. But with the convergence of the state quantity $x$, it will gradually approach the range of $|x| \leq|w| / k$, which leads to the suppression of the disturbance of the system and the steady-state error of the system.

The ADRC reduces the steady-state error of the system by adopting appropriate nonlinear functions. According to the different error states, the nonlinear function can make the feedback gain appropriate increase and decrease, so as to reduce the steady-state error of the system, and effectively avoid the disadvantages caused by adopting the fixed feedback gain.
Nonlinear feedback is used to control objects:

$$
u=-k|x|^{\alpha} \operatorname{sign}(x), \quad 0<\alpha<1 .
$$

Then, the controlled object is converted into a closed loop:

$$
\dot{x}=w(x, t)-k|x|^{\alpha} \operatorname{sign}(x) .
$$

Then get

$\frac{1}{1+\alpha} \frac{d|x|^{1+\alpha}}{d t}=-k\left(|x|^{\alpha} \operatorname{sign}(x)-\frac{w(x, t)}{2 k}\right)^{2}+k\left(\frac{w(x, t)}{2 k}\right)^{2}$.

When $d|x|^{1+\alpha} / d t<0$, the state $x$ is convergent, which requires

$$
|x|^{\alpha} \operatorname{sign}(x)-\frac{w(x, t)}{2 k}>\frac{w(x, t)}{2 k} .
$$

Then get

$$
|x|>\left(\frac{|w(x, t)|}{k}\right)^{1 / \alpha}
$$

According to the analysis of the upper form, when $|x|>$ $(|w(x, t)| / k)^{1 / \alpha}$, the state $x$ converges, and with the further convergence, state $x$ can converge to the range of $|x| \leq$ $(|w(x, t)| / k)^{1 / \alpha}$, so the steady-state error of the system will not be greater than $(|w(x, t)| / k)^{1 / \alpha}$.

It is easier to know the linear feedback obtained the maximum steady-state error $|w(x, t)| / k$ compared with $(|w(x, t)| / k)^{1 / \alpha}$ that when $0<\alpha<1$ and $k>w(x, t)$, there are

$$
\left(\frac{|w(x, t)|}{k}\right)^{1 / \alpha} \ll \frac{|w(x, t)|}{k} .
$$

For systems subject to uncertainties, the steady-state error obtained by using nonlinear feedback is much smaller than that by using linear feedback. In addition, in nonlinear feedback, if the $\alpha$ value is changed slightly, the $(|w(x, t)| / k)^{1 / \alpha}$ can rapidly approach zero.

The TD generates the arranged transition process and its derivatives. The outputs of ESO estimate the states of the control system. By comparing the difference between the outputs of TD and those of ESO, the NLSEF control law $u_{0}(t)$ is used to drive the state trajectory to the desired reference signal. Its mathematic expression is given as [24]

$$
\varepsilon_{i}=v_{i}-z_{i} \quad(i=1,2, \ldots, n) .
$$

Thus, the nonlinear combination of system state error feedback is formed:

$$
u_{0}(t)=\sum_{i=1}^{n} \beta_{i} f a l\left(\varepsilon_{i}, \alpha_{i}, \delta\right)
$$

where $\alpha_{i}, \beta_{i}(i=1,2, \ldots, n)$, and $\delta$ are adjustable parameters. 


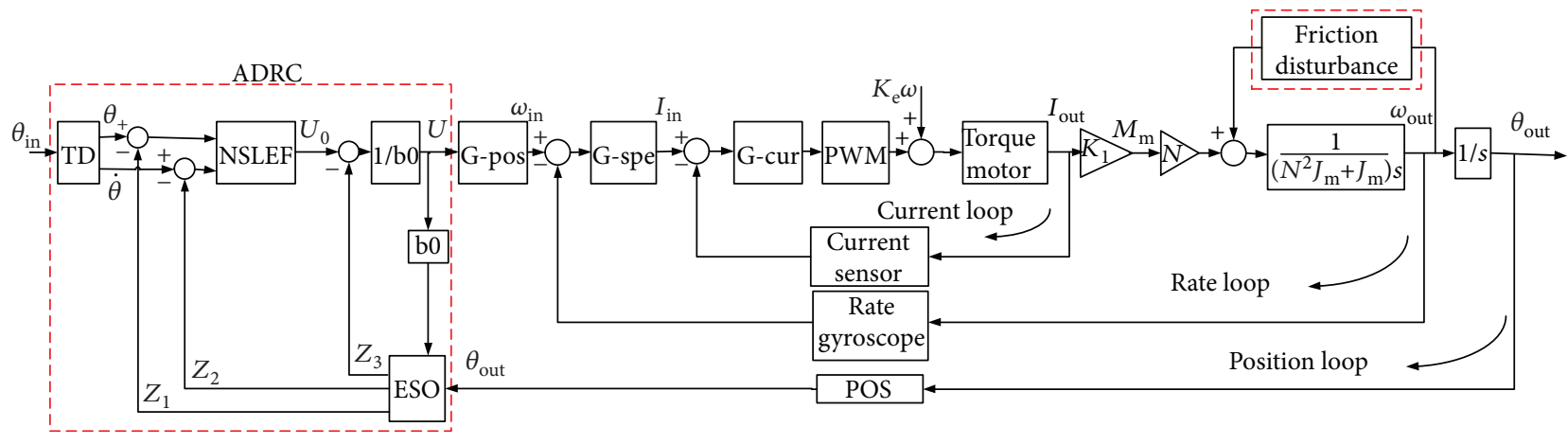

FIGURE 4: Block diagram of the ADRC-based three-loop compound controller.

$$
f a l(e)= \begin{cases}|e|^{\alpha} \operatorname{sign}(e), & |e|>c, \\ \frac{e}{c^{1-\alpha}}, & |e| \leq c,\end{cases}
$$

where $f a l(e)$ is defined as a modified exponential gain function [25].

3.3. Extended State Observer (ESO). The performance of the ESO is very important to the performance of the controller $[18,20]$. The state observer is used to determine the internal state of the system by observing the external state of the system in different situations.

If the function $f\left(x_{1}, x_{2}\right)$ is not known and the above description is not valid, the way to expand the state observer is to expand $f\left(x_{1}, x_{2}\right)$ into a new state variable $x_{3}$ :

$$
\begin{aligned}
& x_{3}=f\left(x_{1}(t), x_{2}(t)\right), \\
& \dot{x}_{3}=w(t) .
\end{aligned}
$$

In this way, nonlinear objects can be regarded as new linear objects [25]:

$$
\begin{aligned}
& \dot{x}_{1}=x_{2}, \\
& \dot{x}_{2}=x_{3}+b u, \\
& \dot{x}_{3}=w(t), \\
& y=x_{1} .
\end{aligned}
$$

Then, the state observer is established:

$$
\begin{aligned}
& e_{1}=z_{1}-y, \\
& \dot{z}_{1}=z_{2}-\beta_{01} e_{1}, \\
& \dot{z}_{2}=z_{3}-\beta_{02}\left|e_{1}\right|^{1 / 2} \operatorname{sign}\left(e_{1}\right)+b u, \\
& \dot{z}_{3}=-\beta_{03}\left|e_{1}\right|^{1 / 4} \operatorname{sign}\left(e_{1}\right) .
\end{aligned}
$$

In this way, the provided parameters $\beta_{01}, \beta_{02}$, and $\beta_{03}$ are selected properly, the state variables of the system can be estimated in real time by $z_{1}, z_{2}$, and $z_{3}$, and the real-time action amount of the acceleration $f\left(x_{1}, x_{2}\right)$ of the system is measured. Equation (25) can not only achieve good control effect but also avoid high-frequency tremor.

$$
\mathrm{fal}(e, \alpha, \delta)= \begin{cases}\frac{e}{\delta^{\alpha-1}}, & |e| \leq \delta, \\ |e|^{\alpha} \operatorname{sign}(e), & |e|>\delta,\end{cases}
$$

where $\delta$ is the length of the linear section.

$$
\begin{aligned}
& z_{1}(t) \longrightarrow x_{1}(t), \\
& z_{2}(t) \longrightarrow x_{2}(t), \\
& z_{3}(t) \longrightarrow f\left(x_{1}(t), x_{2}(t)\right) .
\end{aligned}
$$

If $f\left(x_{1}, x_{2}\right)$ contains time $t$ and unknown disturbances $w(t)$, it is available [17]:

$$
z_{3} \longrightarrow x_{3}=f\left[x_{1}(t), x_{2}(t), w(t)\right]
$$

The real-time action of acceleration acting on the system can be obtained:

$$
a(t)=f\left[x_{1}(t), x_{2}(t), t, w(t)\right] .
$$

Thus, (28) can become the extended state observer of the system. The ESO is a dynamic process that does not take advantage of system parameters and specific models but uses the information between inputs and outputs of the system to estimate the system state variables directly.

3.4. The ADRC-Based Three-Loop Compound Controller. Figure 4 shows the block diagram of the ADRC-based three-loop compound controller. As shown in this figure, the blocks of G-cur, G-spe, and G-pos indicate the controllers from the inner loop to the outer loop, which are the current loop, rate loop, and position loop, respectively; the PWM block indicates the pulse width modulation, which is used for the current amplification to drive the torque motor; the symbol $L, R, K_{\mathrm{t}}$, and $N$ are the inductance of a torque motor, resistance, motor torque coefficient, and transition ratio from the torque motor to the gimbal, respectively. Besides, $J_{\mathrm{m}}$, and $J_{\mathrm{t}}$ indicate the moment of inertia of the motor and the moment of the gimbal inertia along the rotation axis, respectively.

As shown in this figure, in order to improve the control accuracy of the three-axis ISP system, the ADRC controller 


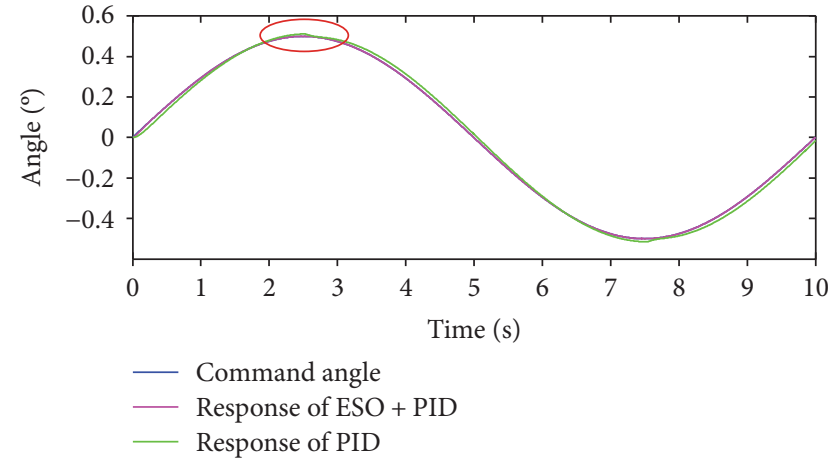

(a) Global curve

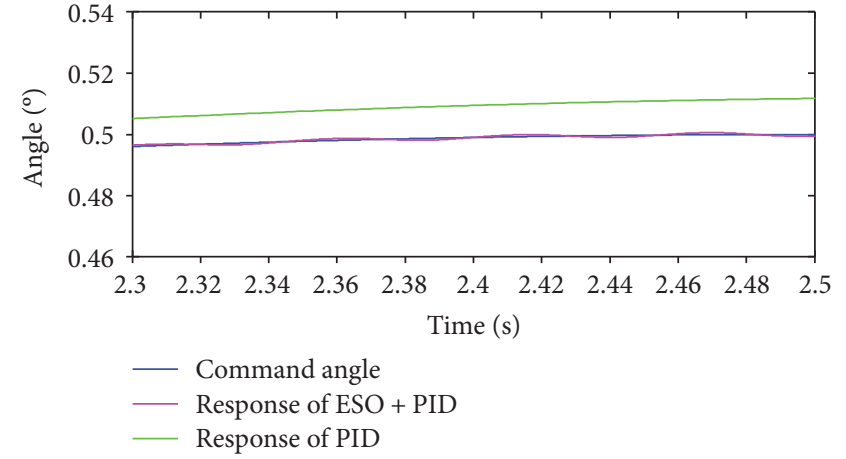

(b) Local magnification curve

FIGURE 5: PID and ESO/PD sine angle position feedback contrast diagram.

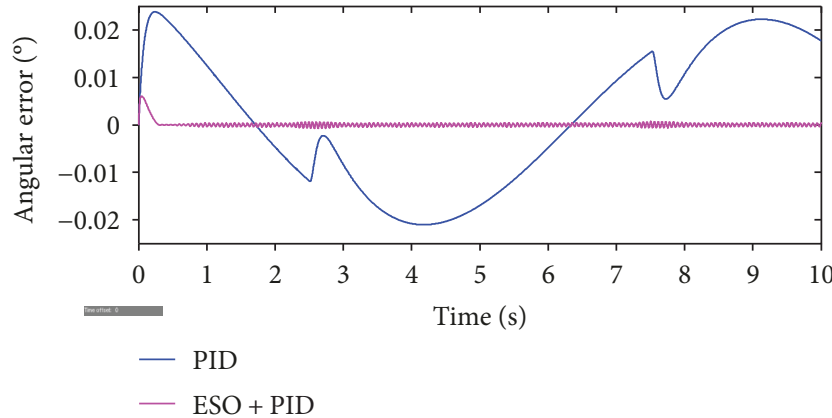

FIGURE 6: PID and ESO/PD angle position error contrast diagram.

is designed. One of the inputs of the ADRC is the tracking instruction of angular position of the ISP control system, $\theta_{\text {in }}$, and another input of the ADRC, $\theta_{\text {out }}$, is the real angular position of the ISP measured by the POS. The control amount $u$ calculated by the ADRC is acted on the ISP position loop.

In Figure 4 , the generalized inputs, $\theta$ and $\dot{\theta}$, are first obtained by the TD. The generalized outputs of the ISP control system, $z_{1}$ and $z_{2}$, are obtained by the ESO. $z_{1}$ is the angle value measured by POS in the position loop of the three-axis ISP system, $z_{2}$ is the derivative of the angle, and $z_{3}$ is the compensatory amount of the ESO to the disturbance in the ISP control system. The compared errors between the generalized inputs and the generalized outputs are inputted to the NLSEF to generate the control amount $u_{0}$, which are compared with $z_{3}$ to generate the control amount $u$. The control amount $u$ after compensation is acted on the position loop of the ISP. Thus, the disturbance rejection ability and the control accuracy of the ISP control system are improved.

\section{Simulation Analysis}

4.1. Angular Position Response to Step Input. In order to verify the validity of ADRC algorithm, MATLAB/Simulink simulation is firstly carried out. The main parameters of the NLSEF control law are $\left\{\beta_{1}, \beta_{2}, \alpha_{3}, \alpha_{4}, \delta_{2}\right\}$. Normally, the value of $\alpha_{3}$ is from 0.5 to 0.75 , and the value of $\alpha_{4}$ is from 1.25 to 1.5 . The value of $\delta_{2}$ is the same as the integral step

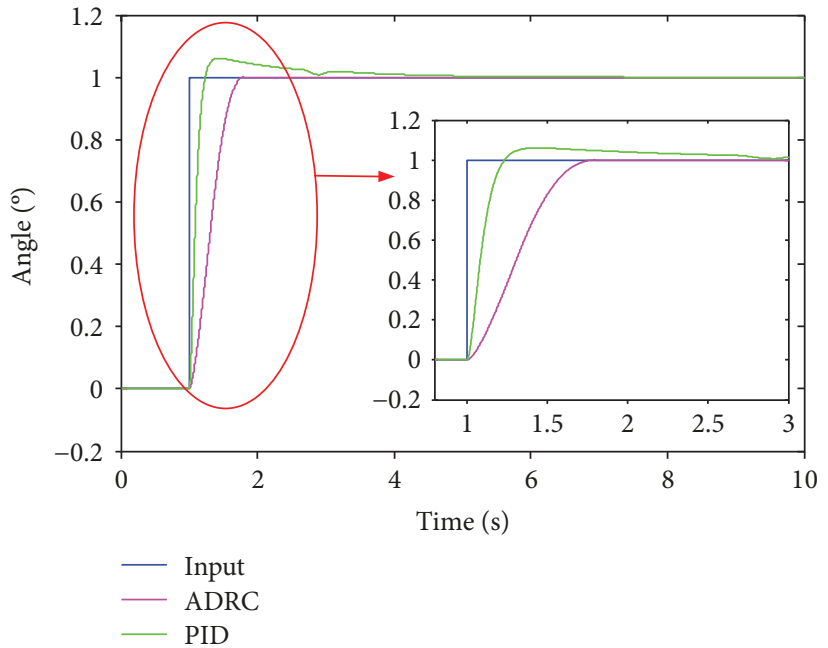

Figure 7: Comparison of position feedback between step angle of conventional PID and ADRC controller.

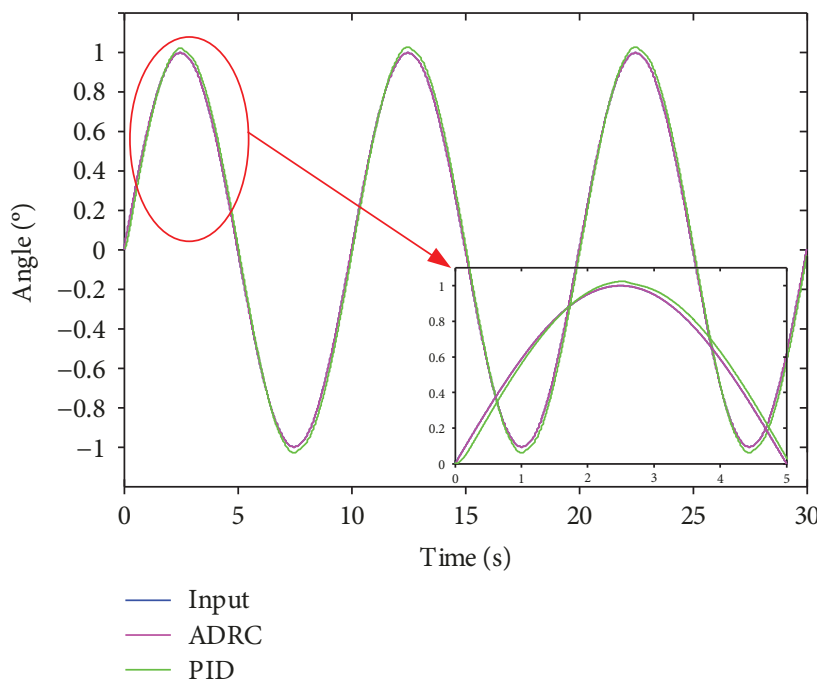

Figure 8: Comparison of position feedback to sinusoidal signal between conventional PID and ADRC controller. 


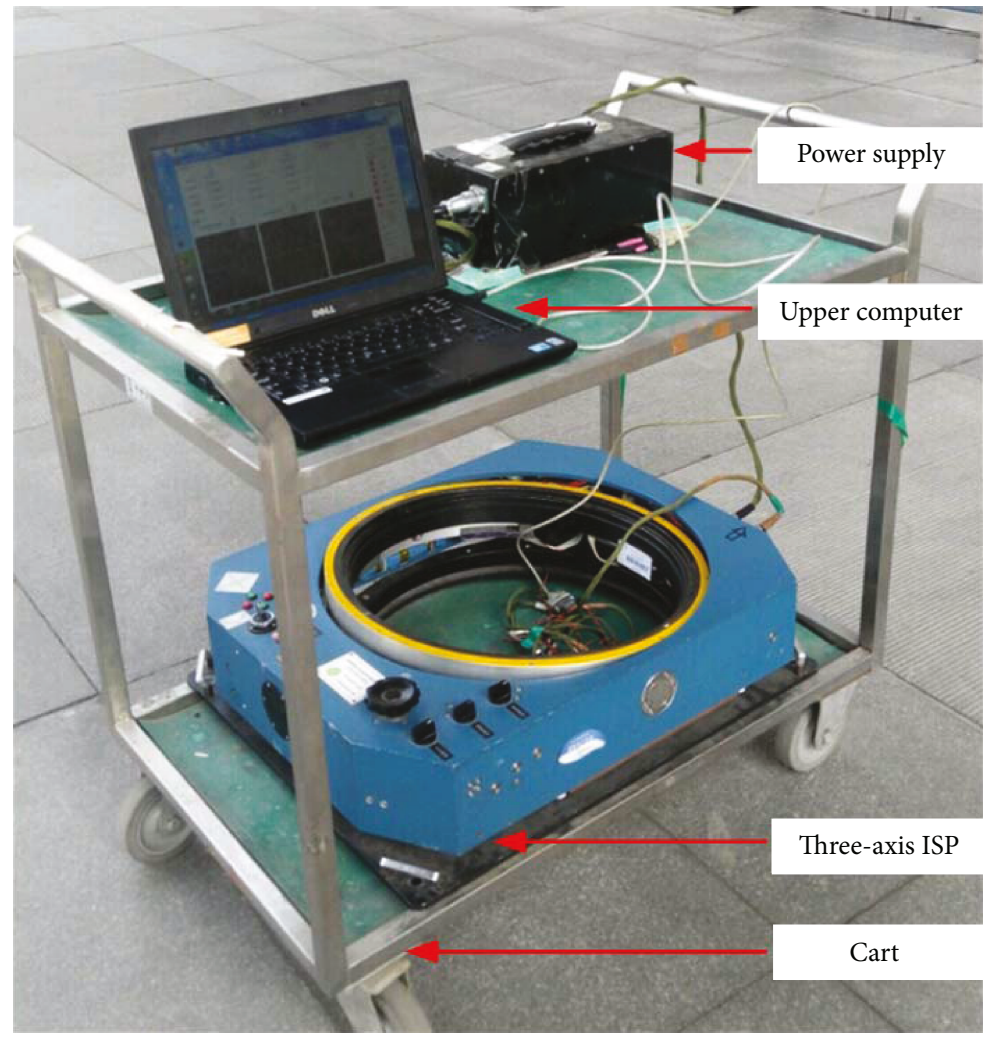

Figure 9: Picture of moving base experiment system.

length as 0.01 generally. The parameters tuned by the experiential method are as follows: $\beta_{1}=6.0$ and $\beta_{2}=1.5$.

In the simulation, the pitch gimbal system is chosen as the control object. When the given input angle instruction is $\theta_{\text {id }}=0.5 \sin \left(\pi \cdot n \cdot T_{\mathrm{s}}\right)$ deg, $T_{\mathrm{d}}=0$, the disturbance resistance performance of the PID and the ESO/PD controller is simulated by adding the disturbance model; the results are shown in Figure 5.

In Figure 5(a), the PID and the ESO/PD composite controllers can track the sinusoidal signals in different position precision. In the local magnification diagram of Figure 5(b), we can see that the PID has approximately $0.01^{\circ}$ errors in tracking the sinusoidal instruction, but the ESO/PD compound controller tracking curve almost coincided with the sinusoidal instruction. Therefore, the ESO/PD controller has strong disturbance resistance ability, which can effectively suppress the disturbance effect, and its tracking error is almost zero.

Under the influence of disturbance, the comparison of angular position error between the PID and the ESO/PD controller is shown in Figure 6. From Figure 6, we can see that the angular error of PID fluctuates slowly from $-0.02^{\circ}$ to $0.025^{\circ}$. When the disturbance amplitude is large, the tracking error curve of the PID will increase correspondingly, while the angular error of the ESO/PD composite controller is stable at $0^{\circ}$. Therefore, the ESO/PD controller has better anti-interference ability than the PID controller.

Figure 7 shows the comparison of position feedback to step angle between conventional PID and ADRC controller.
When the track reference is a step signal with magnitude $1^{\circ}$ which lasts for $10 \mathrm{~s}$, the ADRC controller has a good dynamic performance compared to the conventional PID controller. As shown in this figure, compared to the conventional PID controller, the ADRC controller has nearly no any overshoot and can more easily reach stabilization state with much shorter adjusting time.

\subsection{Angular Position Response to Sinusoidal Input. Figure 8} shows the comparison of position feedback to sinusoidal signal between conventional PID and ADRC controller. When the track reference is sinusoidal signal with amplitude $1^{\circ}$ and frequency $0.1 \mathrm{~Hz}$, the ADRC controller can achieve better disturbance rejection performance compared to the conventional PID controller. As shown in this figure, compared to the conventional PID controller, the tracking curve of the ADRC controller coincides almost with the sine command.

\section{Experimental Verification}

In order to verify the validity and practicability of the ADRC, the experiments are carried out. Figure 9 shows the picture of moving base experiment system. In the experiments, the three-axis ISP is placed on a moving cart. The experimental system includes a three-axis ISP, a data acquisition and processing system (upper computer), and a $28 \mathrm{~V}$ DC power supply. The weight of the ISP is $40 \mathrm{~kg}$ and its maximum support load is $80 \mathrm{~kg}$. The maximum leveling rotation 


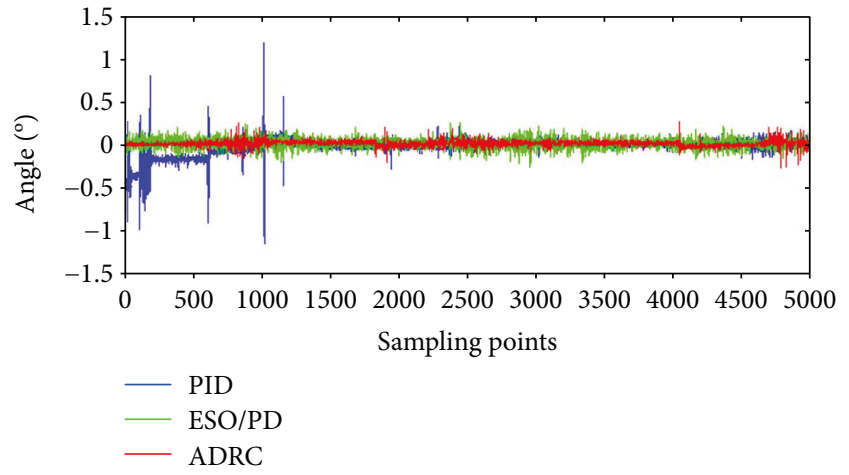

(a) Global curve

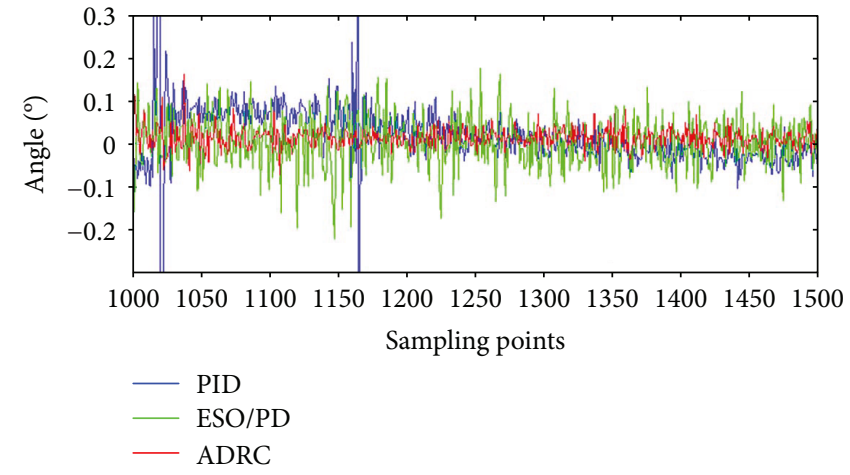

(b) Local enlarged drawing

Figure 10: Comparison of angle error of dynamic cart experiment.

angle range of the ISP is $\pm 5^{\circ}$; the maximum heading rotation angle range is $\pm 25^{\circ}$.

Set the tracking reference angle of the roll gimbal system as $0^{\circ}$; sampling points is 5000 , and the sampling frequency is $50 \mathrm{~Hz}$. As shown in Figure 10, in leveling experiments, the results obtained by three different methods are as follows: for the PID controller, the RMS (root mean square) error is $0.2673^{\circ}$; for the ESO/PD controller, the RMS error is $0.1315^{\circ}$, which is decreased up to $50.8 \%$ compared to the PID controller; for the ADRC controller, the RMS error is $0.0826^{\circ}$, which are decreased up to $69.1 \%$ and $37.2 \%$ compared to the PID controller and the ESO/PD controller, respectively. The results show that the ADRC control method has better control precision and interference suppression capability compared to the PID and the ESO/PD.

\section{Conclusion}

This paper investigates the high-precision control method for a three-axis inertially stabilized platform (ISP). Based on the analysis, a disturbance rejection control scheme based on the active disturbance rejection control (ADRC) is proposed to realize high-precision position servo control, which is robust to the multisource disturbances. Then, the numerical simulations and the experimental validations are conducted to analyze and verify the scheme, respectively. The results show that the ADRC control method can get a better control accuracy and have strong disturbance rejection ability than the conventional PID methods. Under the moving base experimental condition, the stabilization precision of the ISP obtained by the ADRC controller is improved up to $69.1 \%$ compared to the conventional PID control method.

\section{Conflicts of Interest}

The authors declare that there is no conflict of interest regarding the publication of this paper.

\section{Acknowledgments}

This project is supported in part by the National Natural Science Foundation of China (Grant nos. 51775017, 51375036), by the Beijing Natural Science Foundation
(Grant no. 3182021), and by the Open Research Fund of the State Key Laboratory for Manufacturing Systems Engineering (sklms2018005).

\section{References}

[1] M. K. Masten, "Inertially stabilized platforms for optical imaging systems," IEEE Control Systems Magazine, vol. 28, no. 1, pp. 47-64, 2008.

[2] Y. Han, Y. Lu, and H. Qiu, "An improved control scheme of gyro stabilization electro-optical platform," in 2007 IEEE International Conference on Control and Automation, pp. 346-351, Guangzhou, China, 2007.

[3] W. Ji, Q. Li, and B. Xu, "Design study of adaptive fuzzy PID controller for LOS stabilized system," in Sixth International Conference on Intelligent Systems Design and Applications, pp. 336-341, Jinan, China, 2006.

[4] X. Zhou, Y. Jia, Q. Zhao, and R. Yu, "Experimental validation of a compound control scheme for a two-axis inertially stabilized platform with multi-sensors in an unmanned helicopter-based airborne power line inspection system," Sensors, vol. 16, no. 3, p. 366, 2016.

[5] X. Zhou, H. Zhang, and R. Yu, "Decoupling control for twoaxis inertially stabilized platform based on an inverse system and internal model control," Mechatronics, vol. 24, no. 8, pp. 1203-1213, 2014.

[6] Q. Mu, G. Liu, M. Zhong, and Z. Chu, "Imbalance torque compensation for three-axis inertially stabilized platform using acceleration feedforward," in 2012 8th IEEE International Symposium on Instrumentation and Control Technology (ISICT) Proceedings, pp. 157-160, London, UK, 2012.

[7] Z. Li, D. Fan, and S. Fan, "LuGre-Model-based friction compensation in direct-drive inertially stabilized platforms," IFAC Proceedings Volumes, vol. 46, no. 5, pp. 636-642, 2013.

[8] X. Zhou, C. Yang, and T. Cai, "A model reference adaptive control/PID compound scheme on disturbance rejection for an aerial inertially stabilized platform," Journal of Sensors, vol. 2016, Article ID 7964727, 11 pages, 2016.

[9] W. Xue and Y. Huang, "Comparison of the DOB based control, a special kind of PID control and ADRC," in Proceedings of the 2011 American Control Conference, pp. 4373-4379, San Francisco, CA, USA, 2011.

[10] W. Xue, W. Bai, S. Yang, K. Song, Y. Huang, and H. Xie, "ADRC with adaptive extended state observer and its application to air-fuel ratio control in gasoline engines," IEEE 
Transactions on Industrial Electronics, vol. 62, no. 9, pp. 58475857, 2015.

[11] Y. Huang and J. Han, "Analysis and design for the second order nonlinear continuous extended states observer," Chinese Science Bulletin, vol. 45, no. 21, pp. 1938-1944, 2000.

[12] Y. X. Su, C. H. Zheng, and B. Y. Duan, "Automatic disturbances rejection controller for precise motion control of permanent-magnet synchronous motors," IEEE Transactions on Industrial Electronics, vol. 52, no. 3, pp. 814-823, 2005.

[13] W. Xue and Y. Huang, "On performance analysis of ADRC for a class of MIMO lower-triangular nonlinear uncertain systems," ISA Transactions, vol. 53, no. 4, pp. 955-962, 2014.

[14] Q. Zheng, L. Dong, D. H. Lee, and Z. Gao, "Active disturbance rejection control for MEMS gyroscopes," IEEE Transactions on Control Systems Technology, vol. 17, no. 6, pp. 1432-1438, 2009.

[15] S. E. Talole, J. P. Kolhe, and S. B. Phadke, "Extendedstate-observer-based control of flexible-joint system with experimental validation," IEEE Transactions on Industrial Electronics, vol. 57, no. 4, pp. 1411-1419, 2010.

[16] C. Zhao and Y. Huang, "ADRC based input disturbance rejection for minimum-phase plants with unknown orders and/or uncertain relative degrees," Journal of Systems Science and Complexity, vol. 25, no. 4, pp. 625-640, 2012.

[17] S. Li, X. Yang, and D. Yang, "Active disturbance rejection control for high pointing accuracy and rotation speed," Automatica, vol. 45, no. 8, pp. 1854-1860, 2009.

[18] Y. Tang, Y. Wu, M. Wu, X. Hu, and L. Shen, "Nonlinear tracking-differentiator for velocity determination using carrier phase measurements," IEEE Journal of Selected Topics in Signal Processing, vol. 3, no. 4, pp. 716-725, 2009.

[19] P. Wang and K. Zhang, "Research on line-of-sight rate extraction of strapdown seeker," in Proceedings of the 33rd Chinese Control Conference, pp. 859-863, Nanjing, China, 2014.

[20] I. Kasim and W. Riyadh, "On the improved nonlinear tracking differentiator based nonlinear PID controller design," International Journal of Advanced Computer Science and Applications, vol. 7, no. 10, 2016.

[21] H. Huang, L. Wu, J. Han, G. Feng, and Y. Lin, “A new synthesis method for unit coordinated control system in thermal power plant - ADRC control scheme," in 2004 International Conference on Power System Technology, 2004. PowerCon 2004, pp. 133-138, Singapore, 2004.

[22] J. Han, "Nonlinear state error feedback control law-NLSEF," Control and Decision, vol. 3, 1995.

[23] J. Han, "From PID technique to active disturbances rejection control technique," Control Engineering of China, vol. 9, pp. 13-18, 2002.

[24] W. Jun, Y. J. Wang, and H. Jian, "Artificial bee colony algorithm based auto-disturbance rejection control for rehabilitation robotic arm driven by PM-TS actuator," in 2012 Proceedings of International Conference on Modelling, Identification and Control, pp. 802-807, Wuhan, Hubei, China, 2012.

[25] Y. Bing, Z. H. Tian, and S. J. Shi, "Novel fault diagnosis scheme for HVDC system via ESO," High Voltage Engineering, vol. 11, 2007. 


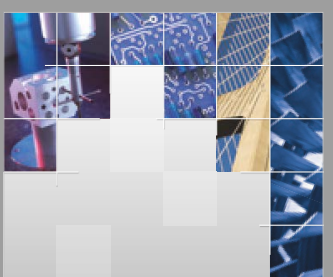

\section{Enfincering}
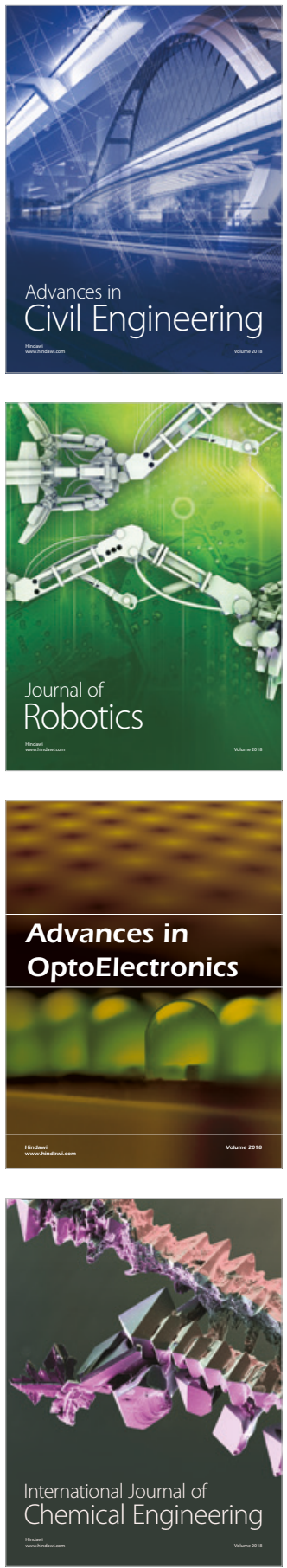

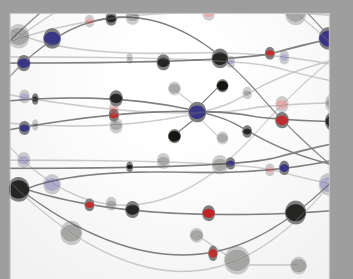

\section{Rotating \\ Machinery}

The Scientific World Journal

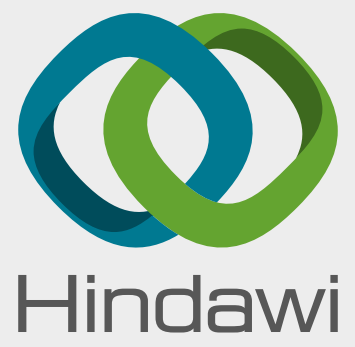

Submit your manuscripts at

www.hindawi.com
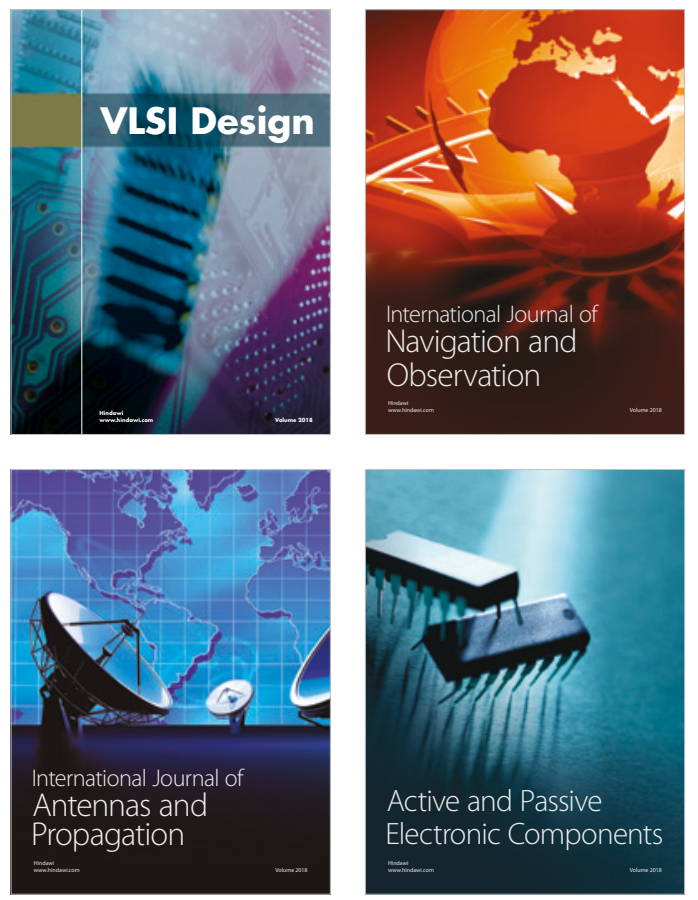
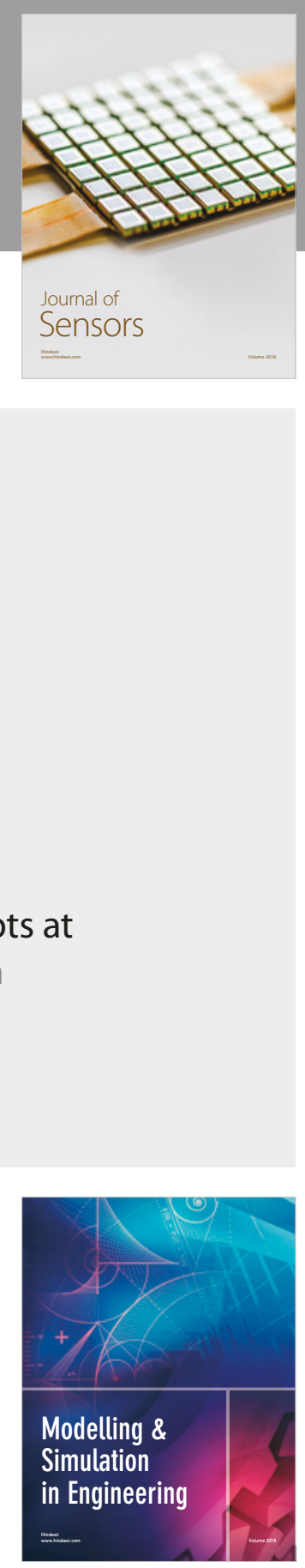

\section{Advances \\ Multimedia}
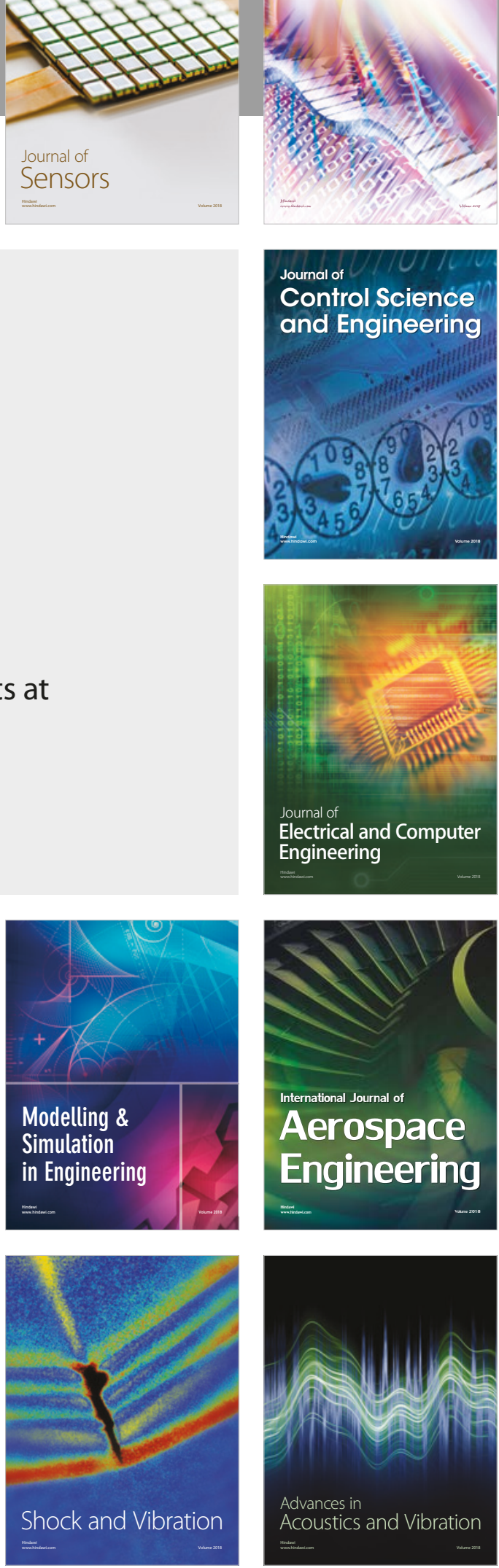